\title{
Configuration du champ de la Communication Organisationnelle au Brésil : problématisation, possibilités et potentialités
}

Configuration of the Brazilian organizational communication field: problematisation, possibilities and potentialities

Ângela Marques et Ivone de Lourdes Oliveira

\section{OpenEdition} Journals

Édition électronique

URL : http://journals.openedition.org/communicationorganisation/5092

DOI : 10.4000/communicationorganisation.5092

ISSN : 1775-3546

Éditeur

Presses universitaires de Bordeaux

\section{Édition imprimée}

Date de publication : 1 décembre 2015

Pagination : 109-124

ISSN : 1168-5549

Référence électronique

Ângela Marques et Ivone de Lourdes Oliveira, « Configuration du champ de la Communication Organisationnelle au Brésil : problématisation, possibilités et potentialités », Communication et organisation [En ligne], 48 | 2015, mis en ligne le 01 décembre 2018, consulté le 04 janvier 2020. URL http://journals.openedition.org/communicationorganisation/5092; DOI : 10.4000/ communicationorganisation.5092 


\title{
Configuration du champ de la Communication Organisationnelle au Brésil : problématisation, possibilités et potentialités
}

\author{
Ângela Marques' et Ivone de Lourdes Oliveira²
}

\section{Introduction}

Lorigine de la Communication Organisationnelle (CO) au Brésil est liée au développement économique, social et politique du pays et également à l'évolution des activités de Relations Publiques (RP) et du journalisme d'entreprise (Kunsch 2008 : 173). Ces secteurs de la Science de la Communication commencent à s'enraciner dans la décennie des années cinquante, avec l'industrialisation promue par le président Getúlio Vargas et les investissements de leur successeur, Juscelino Kubitschek. En 1952, apparaît la première société de communication du pays, la Compagnie Nationale de Relations Publiques et Propagande, qui propose, de manière globale et pionnière, des prestations de services en communication aux entreprises. La décennie des années soixante voit l'expansion des départements de RP et de relations industrielles dans les grandes multinationales (Kunsch 2008), qui ont apporté au Brésil un savoir-faire acquis dans leurs pays d'origine. A partir de la fin des années soixante-dix, les départements gouvernementaux de Relations Publiques et de relations avec la presse, ainsi que ceux de journalisme d'entreprise (focalisé uniquement sur les journaux, revues, bulletins, etc.) se sont développés en s'ajustant aux nouvelles exigences de la $\mathrm{CO}$.

Ainsi, insérée dans le champ des Sciences Sociales Appliquées et des Sciences de la Communication, la $\mathrm{CO}$ se développe-t-elle au Brésil à partir

1 Docteure en communication sociale de l'Universidade Federal de Minas Gerais (UFMG) Belo Horizonte. Post-docteure en Sciences de la communition à l'Université Stendhal, Grenoble III (GRESEC). Professeure du Programme de Post-Graduation en communication à l'UFMG. Ses recherches portent sur la délibération publique, la conversation civique au quotidien et les processus de formation des sphères publiques médiatisées ; angelasalgueiro@gmail.com.

2 Docteure en communication sociale de l'Université Catholique de Minas Gerais (PUC-Minas) Belo Horizonte. Post-docteure en Sciences de l'information et de la communication à l'Université Paul Sabatier Toulouse 3. Professeure en charge du Programme de Post-Graduation en communication sociale de l'Université PUC-Minas. Chercheuse en Communication Organisationnelle ; ivonepucmg@gmail.com 
des Relations Publiques ${ }^{3}$, et en se fondant sur la qualification professionnelle correspondante, notamment grâce aux cours dispensés, depuis 1964, dans plusieurs Universités.

Mais ce processus de construction conceptuelle du champ a postérieurement suscité quelques problèmes, notamment en termes de construction épistémologique.

$\mathrm{Si}$, dans le présent article, seront évoquées quelques-unes des difficultés que rencontrent les études en Communication Organisationnelle, nous souhaitons également pointer également les principales potentialités qui s'offrent aux chercheurs qui œuvrent actuellement à la consolidation du champ, possibilités dont témoignent les recherches actuellement menées dans nos Universités, laboratoires et groupes de recherche.

Citons, dans un premier temps, les principales difficultés, sur lesquelles nous reviendrons ensuite. La première est liée à la relation existant entre la Communication Organisationnelle (CO) et les Relations Publiques (RP). La deuxième concerne les tentatives de construction d'un corps conceptuel, cohérent et consistant, encore en émergence. Le troisième obstacle a trait à l'absence d'une problématisation scientifique intégrant la perspective critique, prenant en compte l'articulation des notions telles que politique, éthique, dialogue, conflits moraux, souffrance sociale, identité, discours, pouvoir et/ou pratiques de résistance. Nous y reviendrons.

Néanmoins, ces trois problèmes, car il s'agit bien de problèmes, n'arrivent pas à neutraliser les innombrables possibilités de croissance de ce champ (cf. en bibliographie, la richesse et la variété des recherches menées) qui porte désormais sur la compréhension des organisations en tant qu'acteurs politiques, en considérant leurs employés comme des agents autonomes et sensibles, qui s'attache à la construction d'une perspective théorique et méthodologique montrant la production de sens dans les organisations (notamment nécessaire pour résoudre les conflits) et qui se préocuppe, in fine, de contribuer à la consolidation des fondements épistémologiques de ce champ d'études.

\footnotetext{
3 Les Relations Publiques comprennent l'ensemble des méthodes (événementiel, relations-presse, etc.) et des techniques utilisées par des entreprises ou organisations (syndicats, partis politiques, États) et/ou par des groupements d'intérêts (lobbying), pour informer le public de leurs réalisations, promouvoir leur image, favoriser la confiance, susciter de la sympathie à leur égard à l'extérieur et favoriser les bonnes relations à l'intérieur.

Au Brésil, les « Relations Publiques » concernent les relations avec les publics au sens large, entendues comme les relations professionnelles (non privées) avec toutes les parties-prenantes, tant du monde des affaires que celui des institutions ou des citoyens.

En France et dans les pays francophones, les Relations Publiques s'intitulent « relations publics » depuis 2011 (cf. Syntec RP, organisme qui défend les intérêts de la profession en France) en vue de clarifier leur champ d'action.
} 


\section{Communication Organisationnelle et Relations Publiques : construction d'un corps théorique et conceptuel}

Dans ce contexte brésilien incertain, l'un des premiers problèmes que doit affronter le processus de consolidation des études de Communication Organisationnelle $(\mathrm{CO})$ est l'existence d'une confusion entre les activités et approches caractéristiques des Relations Publiques et celles de la CO. Initialement nous pouvons indiquer l'existence de différences marquantes entre ces deux champs, leurs fondements théoriques et intentions.

Les Relations Publiques et la Communication Organisationnelle partagent le même espace, les mêmes objets, recherchent des résultats similaires, mais utilisent des méthodologies et fondements théoriques différents, sinon dissemblables. Les RP possèdent certes une épistémologie bien documentée, basée sur les théories des penseurs classiques et contemporains de l'administration d'entreprises et de la communication complétées par des exercices logiques (Iasbeck 2009 : 104-105). Mais il y a toutefois une distinction entre les deux champs d'études : la $\mathrm{CO}$ se préoccupe de la construction réflexive et épistémologique de la communication, alors que les $\mathrm{RP}$ jouent plutôt un rôle de gestion des différentes activités relationnelles dans le contexte des organisations. Or, la distinction entre théorie et pratique est néfaste, nous le savons, et aboutit généralement à des erreurs et à des jugements partiels. Elle contribue à ériger des limites et des frontières poreuses, sans distinctions claires... alors même que les imbrications et les tensions concernées demanderaient plutôt des articulations.

Lune des grosses différences entre la Communication Organisationnelle et les Relations Publiques se trouve dans la nature de leurs concepts : la première est une pensée communicative, une abstraction nécessaire à la compréhension des phénomènes qui, tangibles et évidents, peuvent et doivent être dirigés par la seconde ; quand cette pensée ne s'actualise pas dans des situations maîtrisables, elle peut être uniquement comprise et constituée dans le plan des abstractions et des articulations logiques, dans le flux des idées (Lasbeck 2009 : 106).

Ainsi, que la $\mathrm{CO}$ soit toujours rattachée aux RP fait que la situation au Brésil est différente de celle d'autres pays comme la France, l'Angleterre, le Portugal, la Belgique et les États-Unis, dans lesquels la CO et les RP se configurent plutôt comme différents secteurs de connaissance avec une affiliation historique spécifique de contenus distincts (Rois 2009). Cette interprétation brésilienne occupe en conséquence une place singulière et un regard particulier dans le monde de la connaissance. Malgré le fait que les des deux champs ont la même origine conceptuelle, il n'y a pas encore eu de discussion scientifique sur l'éventuel élargissement du concept de RP, rendant in fine difficile la compréhension des différences entre les corps théorique et conceptuel, entre les pratiques professionnelles de l'un et de l'autre. 
Cependant, des interfaces académiques existent, s'exprimant avantageusement dans la production intellectuelle d'un groupe de chercheurs brésiliens qui travaillent sur la $\mathrm{CO}$ et les $\mathrm{RP}$, et qui, se prévalant d'un dialogue productif entre ces deux versants de la connaissance, contribuent à produire une meilleure compréhension des interactions communicatives dans le contexte des organisations.

En outre, prenant en compte le fait que la réglementation de l'activité de RP au Brésil se produit à la fin de la décennie des années soixante, période du début de la structuration académique du champ (d'ailleurs fortement influencée par les contributions des études managériales et de l'Administration), il est judicieux de mentionner que les réflexions de cette époque ont comme objet les RP considérées comme étant une activité menée en vue d'influencer les relations de travail, qui a mené les entreprises à entretenir une communication optimale avec leurs publics internes et externes.

Je comprends les Relations Publiques comme partie intégrante du sous-système institutionnel des organisations, jouant le rôle fondamental de soigner des Relations Publiques des groupements sociaux qui peuvent être configurés à partir de différentes typologies et caractéristiques structurelles en impliquant des organisations publiques, des sociétés privées et des segments organisés de la société civile (Kunsch 2009 : 54).

L'activité professionnelle émerge ainsi d'une injonction du marché, aboutissant à la production de publications encadrées, et de la nécessité de transmission des informations. Par ce biais, la communication acquiert au fil du temps, de plus en plus de force dans les organisations, à la fois en tant qu'instrument de contrôle et de gestion. La communication jusqu'à alors pratiquée dans l'environnement corporatif en vient à se limiter petit à petit à des questions d'ordre opérationnel, de contrôle et de coercition, et devient in fine un instrument disciplinaire des publics interne et externe.

Depuis les années 1990, avec l'élargissement des études dans le domaine des $\mathrm{RP}$, les études sur la $\mathrm{CO}$ commencent à être réalisées dans une perspective plus générale, mais sont encore traversées par le paradigme classique et informationnel. Néanmoins ses études deviennent légitimes dans le nouveau scénario académique et scientifique du Brésil à une époque où le pays reprend le processus démocratique, et où la société civile brésilienne se réorganise en développant sa force de participation. Au-delà des conditions sociales et économiques du pays, mentionnons certains changements de la société contemporaine qui ont contribué à l'accélération de la présence de ces études au sein de l'académie, spécialement la globalisation qui a provoqué des transformations économiques, culturelles et sociales en obligeant les organisations à redimensionner leur rôle et leur relation avec la société. Ce contexte a amplifié, nous semble-t-il, les possibilités de croissance du champ, mais sans inciter une réflexion approfondie à propos de sa constitution. 
Avec l'ouverture des frontières et le développement d'une société de l'information et de la communication, les organisations adoptent désormais un regard différencié sur leurs politiques et actions respectives. Elles commencent à jouer un rôle de coresponsabilité dans le discours idéal de « la construction d'un monde meilleur » et sont reconnues comme des agents de culture, comme des acteurs sociaux. Citons par exemple, la création à cette même période de l'Institut Ethos avec mission de stimuler les entreprises à gérer leurs affaires de façon socialement responsable.

La communication dans le contexte des organisations a été et est encore fréquemment traitée uniquement comme l'une des plusieurs activités organisationnelles, comme un outil de gestion. Associée souvent à des modèles linéaires d'interaction, la communication est perçue alors comme un moyen de transmettre des informations et des connaissances, un moyen d'influencer les personnes. Une telle approche traditionnelle a pour but la quête du meilleur message à délivrer et la recherche du moyen de contact le plus efficace avec les publics cibles, celui qui permettrait l'imposition d'une manière de penser et/ ou l'incitation à réaliser des tâches déléguées (Colnago 2007).

Selon Kunsch (2009 : 55), la Communication Organisationnelle peut être considérée comme « un phénomène qui se produit dans les organisations avec toute une complexité de processus ", et comme une discipline qui se consacre à étudier le phénomène communicationnel dans le contexte de performance des organisations, en considérant leurs pratiques internes et leurs interfaces avec les dynamiques sociales, économiques et politiques.

La Communication Organisationnelle est la discipline qui étudie comment l'on peut traiter le phénomène communicationnel à l'intérieur des organisations. Elle analyse le système, le fonctionnement et le processus de communication entre l'organisation et leurs divers publics. Elle comprend la communication institutionnelle, la communication commerciale, la communication interne et la communication administrative (Kunsch $2008: 87$ ).

Les RP, à leur tour, figurent comme «secteur appliqué» ${ }^{4}$, en impliquant la planification et la gestion de la communication dans les organisations entretenant des médiations avec différents publics.

Entre les raisons d'être des Relations Publiques, les spécifications dans des fonctions se superposent. Les attributions de cette activité professionnelle sont attachées aux actions de responsabilité dans la génération/production du discours, dans la transmission des informations et dans la vigilance continue de la réception. En d'autres mots, les Relations Publiques s'occupent essentiellement de la qualité du

\footnotetext{
4 Les Relations Publiques « évaluent les comportements institutionnels et des publics, par le moyen de recherches d'opinion et d'auditorats sociaux et d'image ; ils dirigent des perceptions et des Relations Publiques ; en jouant essentiellement quatre fonctions : administrative, stratégique, médiatrice et politique ». «Telle charge implique une série de questions qui impliquent planification, gestion, processus, techniques, instruments, performance de fonctions et activités avec des bases scientifiques et support technique et technologique » (Kunsch 2009, 54-55).
} 
processus de la communication dans les organisations et se distribuent par des secteurs stratégiques dans lesquels il est possible de contrôler, coordonner, superviser, exécuter et évaluer, enfin, diriger la communication. Les professionnels de Relations Publiques sont, donc, directeurs, exécuteurs et inspecteurs de la Communication Organisationnelle (Iasbeck 2009 :105)

La difficulté à définir les différences entre les deux champs est renforcée par la dimension institutionnelle, dans la mesure où une seule revue académique brésilienne réunit la production scientifique et l'association brésilienne de chercheurs utilisant, toutes les deux, le nom de "Communication Organisationnelle et Relations Publiques »(Oliveira 2009). Cela produit une tension supplémentaire dans la littérature brésilienne et dans les débats académiques. Cette situation rend invisible une discussion épistémologique approfondie qui pourrait (nous) aider à comprendre si la question est l'expression de différents savoirs ou seulement celle d'approches distinctes. Or, le manque de clarté des différences et des intersections entre les deux champs peut donner lieu à un blocage dans le processus de construction de la connaissance, en compromettant scientifiquement les champs concernés.

En outre, l'utilisation reliée des deux termes ( $\mathrm{CO}$ et $\mathrm{RP})$ peut encore avoir un sens corporatiste dans la défense de l'habilitation de RP, car la CO devient un champ de recherche et réflexion de plus en plus solide et ramifié. En 2009, Reis discute déjà ainsi ces questions et déclare que des " préoccupations liées au niveau épistémologique ne doivent pas se mélanger avec des efforts de réserve de marché qui finissent pour délimiter un secteur de connaissance à des compétences techniques, en empêchant une approche plus holistique des objets communicationnels » (Reis 2009 : 47).

Nous comprenons la $\mathrm{CO}$ comme un champ de connaissance qui a pour objet l'étude des phénomènes communicationnels dans le contexte organisationnel « en tant que construction conceptuelle pour comprendre les relations construites par et dans les organisations " (Marques 2015 : 7) publiques, privées et non gouvernementales. En ce sens, nous considérons l'organisation comme un acteur social collectif et comme un espace d'interactions conformé par la relation entre des interlocuteurs - c'est-à-dire, qu'elle se constitue comme sujet relationnel qui se construit autour des idées de langage, disputes de pouvoir et de sens (Marques 2015).

Pour accompagner les changements de la société, les organisations commencent à construire leurs pratiques discursives avec la volonté de légitimer leur engagement sur les questions sociales, environnementales et avec leurs employés. La communication est alors revendiquée pour soutenir les efforts de dialogue en favorisant des mécanismes de participation interne et externe. 


\section{Perspectives conceptuelles en Communication Organisationnelle : spécificités et potentialités}

Depuis 2005, la CO développe des bases plus solides et acquiert plus d'espace dans le cercle académique, notamment en raison de la croissance des programmes de post-graduation stricto sensu, de l'investissement en recherche, de la création de la revue Organicom (en 2004) et de l'association nationale de chercheurs (en 2006). Il est maintenant possible de percevoir un changement significatif concernant la forme de concevoir les phénomènes communicationnels dans le contexte des organisations. Les chercheurs sont en quête d'une autre direction épistémologique et s'interrogent à propos du lieu commun qu'elle occupe dans l'Académie, de la dépendance théorique des études organisationnelles et du ton prescripteur caractéristique d'une partie de la littérature encore en vogue. En outre, ces chercheurs interrogent la préférence pour le factuel et le faire, et rendent visibles des questions nouvelles, jugées non pertinentes jusque-là.

Ce contexte a influencé l'approfondissement des discussions sur le caractère stratégique de la communication, sur les pratiques d'agencement communicationnel des organisations, sur la frontière entre les phénomènes de la communication et celui de la construction des organisations, sur la réalité sociale et organisationnelle, ainsi que sur la nécessité de porter un regard multiple sur la complexité des phénomènes communicationnels dans le contexte organisationnel. Cet investissement peut être vu comme une tentative de surmonter

[...] la dichotomie imposée artificiellement entre le professionnel et le théoricien, ce qui place les études de communication dans le contexte des organisations dans un nouveau vecteur, dérivé du commentaire soigneux du quotidien - ensemble d'expériences ponctuelles et définies dans le temps - et d'une théorisation qui échappe au vécu pour produire une expérience, quelque chose qui extrapole le présent exactement pour pouvoir y penser (Pinto $2011: 10$ ).

Plusieurs auteurs brésiliens contemporains développent alors des recherches concernant le champ de la $\mathrm{CO}$ à partir des études de Sociologie, de Politique et/ ou du langage dans le but de la constituer à partir de termes épistémologiques, méthodologiques et théoriques validés (Oliveira, Soares 2008).

Un consensus semble désormais exister entre les divers auteurs : considérer l'organisation comme un phénomène communicationnel continu et même une auto-organisation grâce à l'interaction communicative. Dès lors, la communication est partie intégrante des organisations, qui sont constituées par des personnes qui communiquent et tentent d'atteindre des objectifs organisationnels communs. "Les organisations ne peuvent pas être vues et comprises comme des entités qui existent seulement pour accomplir des objectifs ou des fins spécifiques » (Kunsch 2008 :178). 
Le fait que les organisations soient composées de personnes qui possèdent des univers cognitifs très différents, ainsi que des cultures et points de vue divers implique la complexité des processus communicationnels à considérer (Baldissera, 2008, 2010). Il est nécessaire, en conséquence, de mettre en lumière les aspects relationnels, les contextes, les conditionnements internes et externes, qui marquent les dynamiques de construction de relations sans les réduire à la transmission ou au simple échange de messages.

Ces tensions liées à des asymétries de pouvoir et aux inégalités communicatiionnelles de chacun nous rappellent que le traitement des interactions entre les organisations et leurs publics ne peut être réduit à une analyse des causes et des effets (dans une perspective fonctionnaliste, ou postiviste trop simple). D'un côté, nous ne pouvons pas affirmer que les publics possèdent une forme d'agence libre des contraintes imposées par les pratiques organisationnelles. De l'autre, "les organisations ne doivent pas avoir l'illusion selon laquelle tous ses actes communicatifs aboutissent dans des effets positifs désirés ou qui sont automatiquement acceptés et répondus comme attendu » (Kunsch 2008 : 179).

Les organisations sont des systèmes de construction sociale de significations partagées (Oliveira 2009 ; Baldissera 2010 ; Lima 2008). Elles se configurent par le biais de relations sociales, dans lesquelles la réalité est différemment expérimentée par chacun des membres. L'organisation est un système ouvert, qui interagit avec l'environnement externe de façon continue tout en étant la scène de relations entre des personnes ayant des désirs, des compétences, et des conditions d'exercice différentes.

La construction d'une approche plus relationnelle et critique de la compréhension de la Communication Organisationnelle exige de penser sa dynamique comme un processus relationnel qui n'est pas exempt d'inégalités et d'asymétries, mais pourtant nous place devant une myriade de processus de production de sens et d'expériences, car réunissant des identités personnelles et collectives, elles-mêmes résultantes de processus communicationnels. Dans cette perspective, la préoccupation va de l'expression de significations à la compréhension des processus de communication qui produisent activement du sens, des sentiments, des identités et des expériences du monde (Deetz 2010).

Une relation complexe entre organisations et individus est considérée comme réciproque mais dépendante de facteurs comme le contexte (environnement interne et externe), la structure (normes de relations organisationnelles) et les processus d'échange (comportement de la communication dans les organisations). "Si les organisations sont des processus d'interaction et de relation, il faut mettre en évidence, dans leurs contextes et structures, les pratiques communicationnelles qui incitent ce développement, en comprenant ces réalités comme dynamiques, fluides et activement construites " (Marchiori, 2009 : 11). 
Il ne faut pas considérer la communication comme quelque chose qui se produit uniquement à l'intérieur de l'organisation, mais il faut observer principalement la façon dont l'organisation émerge de la communication. La communication est focalisée à partir d'un contexte conformé par la relation entre les interlocuteurs : l'organisation est perçue en tant que sujet relationnel complexe qui, dans l'interaction avec leurs membres et avec la société, configure un contexte d'interactions. Ainsi, l'approche communicationnelle dans le contexte des organisations s'intéresse à l'analyse du mouvement, en étudiant les manières dont l'interaction actualise la relation entre l'organisation et ses interlocuteurs, en les plaçant et en les replaçant les uns devant les autres pour que des sens soient construits et reconstruits de manière incessante (Oliveira, 2009).

En ce qui concernant l'absence de problématisation de la relation entre " organisation » et " communication » il est possible d'affirmer que nous sommes proches de regarder de façon critique les phénomènes et de considérer les tensions existantes. L'intérêt des chercheurs brésiliens est évident et la majorité d'entre eux s'engage dans la construction épistémologique du champ. La communication dans le contexte des organisations devient une possibilité d'établissement d'échanges et de négociations dans lesquelles la diversité et la tolérance sont présentes.

Il faut prendre en compte que la $\mathrm{CO}$ est un processus de construction et de dispute de sens dans un contexte de relations intersubjectives et, ainsi, que la notion de complexité nous amène à interroger la manière dont les personnes jouent plusieurs rôles sociaux, construisent des identités multiples et s'engagent dans des conversations dans lesquelles ils justifient des points de vue entre des amis, des personnes connues, des collègues de travail ou même des inconnus (Baldissera, 2014).

La communication est une action et aussi un champ de connaissance complexe. Elle est fragmentée, négociée, dialogique, relationnelle, coopérative, conflictuelle et articule les parties et partenaires que s'opposent et se complètent simultanément. Les sujets participants au processus communicationnel, en tant que des "forces en relation, proposent, contestent et internalisent le sens comme des forces actives, réactives, organisatrices, désorganisatrices, complémentaires et/ou antagoniques » (Baldissera 2008 : 167).

Dans cette perspective, la complexité de la relation ordre/désordre dans un processus communicationnel qui apparaît quand nous constatons, dans la pratique, qu'expériences désordonnées et conflictuelles se révèlent nécessaires pour produire des accords, vu que les partenaires d'interlocution s'efforcent, surtout lors de crises et de dilemmes, de se faire comprendre en négociant du sens, des raisons et des informations. 


\section{Communication Organisationnelle : perspective critique}

Il est d'ores et déjà possible d'affirmer que le XXI ${ }^{\mathrm{e}}$ siècle sera marqué par d'autres tendances d'études et d'autres questions de recherche sur la $\mathrm{CO}$ si nous considérons les regards et perspectives en provenance des Sciences Humaines et Sociales (SHS). La littérature scientifique s'intensifie avec la production de livres, d'articles et de séminaires thématiques nationaux et internationaux. Les groupes de recherche proliferrent et jouent un rôle fondamental dans l'articulation des recherches communicatives dans le contexte des organisations.

Malgré le fait que la pratique communicationnelle au Brésil soit encore motivée par le paradigme informationnel, la configuration des phénomènes qui change la société prouve la complexité du processus communicationnel en en faisant le modèle inadéquat pour comprendre l'interaction entre des sujets et la circulation des informations. De cette manière, l'approche critique de la $\mathrm{CO}$ s'intensifie en mettant en évidence le rôle attribué au dialogue dans la résolution de conflits et dans l'établissement de principes éthiques et moraux de comportement. Le dialogue ne peut pas être caractérisé sous sa forme uniquement positive de garantir équité, réciprocité et participation dans les processus argumentatifs qui s'établissent dans les contextes organisationnels. Il est fréquemment utilisé en tant que rhétorique ou même comme " maquillage » d'une communication profondément inégale et asymétrique. "Les organisations défendent le dialogue dans leurs discours, mais ce quelles exigent de leurs employés reflète des structures de pouvoir de caractère très peu démocratique, des structures qui arrivent même à empêcher la vie privée de leurs collaborateurs » (Freitas $2008: 140$ ).

Traiter de la Communication Organisationnelle par une perspective critique exige de considérer non seulement les moyens dont le dialogue est discursivement présenté en tant que pratique et règle de conduite, mais aussi comment s'établissent les manières de convivialité à partir de bases morales et éthiques. En ce sens, le modèle d'interaction communicationnel dialogique se présente comme un locus de discussion éthique, puisqu'il reconnaît la possibilité d'un espace commun de partage de normes, d'intérêts et de décisions publiques qui s'intéressent à tous les acteurs de l'interaction négociée (Oliveira et Paula 2007). " À côté de la discussion morale il faut chercher un processus de démocratisation organisationnelle qui a comme une de ses manifestations plus significatives l'autonomie des individus » (Paula 2005 : 31). Cette démocratisation est considérée comme un réseau de relations traversées par la réflexion éthique des individus, dans sa dimension individuelle et collective.

Un agir gestionnaire et une culture organisationnelle guidés par le discours peut viabiliser le processus de démocratisation du contexte organisationnel et rendre possible la coopération de tous les agents dans la quête de ressources matériels, 
et aussi de ce qui est juste, c'est-à-dire, de ce qui est défini de manière autonome comme justice par une collectivité (Paula 2005 : 41).

La réflexion éthique et morale concernant les pratiques communicatives dans le contexte des organisations met en scène des questions concernant " ce qui est juste pour tous ", dans une dimension collective, et aussi ce qui est défini comme "bien vivre ", dans une dimension plus individuelle et personnelle. Cette réflexion dépend d'actions réglées par la coopération et par la négociation dialogiques, vu que des actions instrumentales (tournées uniquement vers l'accomplissement d'objectifs et la maximisation des performances) dans l'espace organisationnel ont produit des pathologies sociales qui aboutissent à un degré croissant de réification et déshumanisation de l'individu (Baldissera 2014). Cette déshumanisation apparait principalement sous la forme de l'individualisme, de la faillite des liens de solidarité et de l'affaiblissement de gestes d'empathie (Paula 2005). Ce processus amène les individus à affronter les obstacles qui s'opposent à leur autonomie et leur émancipation. L'un de ces obstacles est notamment la souffrance sociale dans le contexte du travail.

Renault (2004) souligne la manière fragmentée et immatérielle de l'organisation du travail dans les sociétés contemporaines et argumente que, d'un côté, l'investissement dans des projets permet de conférer un sens aux principes d'autonomie et de réalisation de soi, mais, de l'autre côté, des hiérarchies invisibles et puissantes prennent la place des relations de subordination des organisations en même temps que des contraintes contrecarrent l'autonomie et l'autoréalisation. La croissance de la précarité du travail est accompagnée de la quête de la flexibilité et de l'individualisation des tâches qui tendent à questionner la relation que le travailleur peut avoir dans une fonction spécifique tout en lui permettant la construction d'une identité professionnelle valorisante.

La fragmentation du travail (passage d'un projet à l'autre, d'un emploi à l'autre) ne permet pas d'intégrer les épisodes de la vie professionnelle dans un récit cohérent, ce qui empêche d'atténuer le durcissement du travail (transformation de la souffrance en plaisir), c'est-à-dire, de valoriser l'investissement psychique lié à l'injonction de l'autonomie et à l'exigence d'investissement total dans le projet (Renault 2004 :226).

Néanmoins, les sujets restent encore dotés d'agence : les processus normatifs et prescripteurs de gestion ne réussissent pas à les subjuguer suffisamment pour les transformer en de simples objets écrasés par les discours stratégiques. Les sujets possèdent des corps où s'inscrivent et se produisent des récits, des émotions et sentiments moraux, éléments qui à tout moment peuvent être actionnés et éclater dans le quotidien organisationnel. C'est à cause de tout cela que, dans une certaine mesure, les processus de constitution des sujets dans les scénarios organisationnels insinuent le dialogue comme contexte dissensuel et polémique, geste d'expression des forces et des idéologies, des 
hostilités et des asymétries, des situations d'abus et de manque de respect, avec une volonté de modelage de nouveaux accords moraux en contextes organisationnels, qui se présentent comme des processus qui tôt ou tard émergeront dans les organisations, au prix de la perte de l'emploi et/ou la dissolution d'un lien formel (Mafra, Marques 2015).

Dans cette perspective, le dialogue peut apparaître comme possibilité de réorganisation de l'espace moral de l'organisation, révélateur du pouvoir politique présent (ou absent) dans les manières de présentation et de représentation des sujets parlants (n'oublions pas, en effet, que le dialogue peut parfois avoir des facettes violentes et agressives). C'est donc à cause de cela que le dialogue, dans la façon dont il se manifeste (occulte ou explicite), révèle des paramètres et des conditions permettant de comprendre les conflits moraux dans les contextes organisationnels en tant que des mécanismes de reconnaissance (ou méconnaissance), de l'empathie ou de la résistance.

La perspective relationnelle est une autre visée qui crée des bases solides pour les réflexions d'ordre théorique et conceptuel ainsi que les recherches empiriques, une fois qu'elle se trouve en harmonie avec l'environnement contemporain de partage symbolique où les sujets sociaux s'interpellent mutuellement. França (2002) propose quelques axes pour consolider la communication dans ce contexte : la communication doit être vue comme un processus d'interaction et de pratique, de production et d'interprétation entre des sujets sociaux, au lieu de confirmer la polarisation entre des émetteurs et des récepteurs. La communication est aussi une réalité où il est possible d'identifier des marques symboliques de ces sujets et de l'environnement qui les enveloppe. La communication est un processus dépendant d'un certain contexte dans lequel se produisent des manifestations et des événements.

Cette visée paradigmatique peut contribuer à provoquer les organisations à regarder les conflits et les divergences en tant que des éléments constitutifs du processus communicationnel. À la fin, à partir du regard de l'autre peuvent apparaître des constatations critiques importantes qui nous conduisent à repenser les stratégies de communication et les relations avec la société (Barros, Brito, Machado 2015).

La perspective relationnelle (Lima 2008) nous montre que la production de sens et la participation des individus à la création de leurs propres environnements sont centrales. Lobjet de la $\mathrm{CO}$ est la communication ellemême en tant que construction conceptuelle caractérisée par des relations construites par et/ou dans les organisations (Colnago 2007). L'expérience personnelle et la procédure de production et négociation de significations sont perçues comme partie fondamentale de pratiques d'interaction plus larges. Les expériences des individus impliquées dans ces pratiques finissent par intervenir dans les significations qui sont produites et qui circulent collectivement. La $\mathrm{CO}$ peut ainsi être vue en tant qu'un processus de construction et de dispute 
de sens, mais aussi comme procédure dialogique, politique et critique qui promeut la configuration de significations, d'informations et de connaissances.

Plusieurs auteurs brésiliens ont adopté le paradigme de la complexité d'Edgar Morin pour comprendre et analyser la communication dans le contexte organisationnel. Ils reconnaissent que l'organisation s'organise dans la perspective d'ordre et de désordre, de moments de collaboration et résistance, d'autonomie et de dépendance, d'antagonismes et de complémentarités. La contribution de ce paradigme est de prouver que nous ne sommes pas submergés dans les certitudes et dans le contrôle de la gestion, mais en coexistence avec les incertitudes de la société et de la dynamique des processus interactionnels et donc devant la connaissance multidisciplinaire qui nous aide à comprendre les diverses dimensions d'un phénomène (Alencar 2015).

Une autre tendance perçue dans la construction de connaissances sur la $\mathrm{CO}$ est l'utilisation des fondements théoriques de la Théorie des Systèmes Sociaux, (Luhmann, Niklas) qui considèrent les organisations en tant qu'un système social.

En ce sens, les organisations se constituent comme des instances opérationnellement fermées, auto-référentes et auto-poétiques dans la mesure où elles entreprennent une série d'efforts pour réduire la complexité de son entourage imprévisible et chaotique, dans une tentative d'auto préservation et évolution. Néanmoins, ce cycle se complète en combinant simultanément l'ouverture et l'interaction avec d'autres systèmes comme la société, l'environnement, la politique et l'économie, entre autres (Mol 2015 : 56).

Nous pouvons actuellement affirmer que la recherche et les études en $\mathrm{CO}$ au Brésil se développent avec l'intention d'établir une révision et de contribuer à une reconstruction du champ en délimitant ses potentialités en tant qu'un savoir de grande importance pour la société. $\mathrm{La} \mathrm{CO}$ requiert donc un regard constitué de bases théoriques d'interface, en privilégiant des « interprétations concernant les processus communicationnels des organisations et leurs frontières avec des paradigmes et des dimensions théoriques d'autres champs de connaissances au-delà des études organisationnelles et administratives traditionnellement perçus comme principales références dans la recherche et la littérature sur la Communication Organisationnelle » (Oliveira, Soares 2008:9).

Il est ainsi possible de conclure que cette construction vise à mettre en question des caractéristiques sédimentées tout au long du processus d'abstraction et de l'interprétation des perspectives théoriques et conceptuelles, en interrogeant l'influence de la pratique professionnelle dans la théorisation des phénomènes communicationnels dans l'environnement organisationnel. La perspective relationnelle souligne la préoccupation du lieu de parole à partir de la communication, la construction de dialogues avec les interlocuteurs en considérant leur expérience historique et personnelle, le questionnement de 
la relation de l'employé avec le monde du travail à partir d'une vision critique et stratégique des processus communicationnels des organisations (et dans les organisations), établie par leurs partenaires, la reconnaissance des pratiques discursives et de la construction de sens dans les processus d'interlocution, la compréhension de l'organisation en tant qu'acteur social, entre autres. Le défi majeur auquel faire face aujourd'hui est lié aux questions méthodologiques qui traversent le champ et qui sont régulièrement dépassées par l'apparition de techniques méthodologiques nouvelles dans des recherches qui ne sont pas uniquement des études de cas configurées de façon descriptive.

\section{BIBLIOGRAPHIE}

ALENCAR Terezinha Gislene R., Possibilidades do paradigma da complexidade na comunicação organizacional, in OLIVEIRA Ivone de L., PENNINI Anice, MOURÃO Isaura, Compreendendo um campo de conhecimento: reflexões epistemológicas sobre a comunicação Organizacional a partir de autores Brasileiros. Curitiba, PR:CRV, 2015.

BALDISSERA Rudimar, "Comunicação organizacional: uma reflexão possível a partir do paradigma da complexidade», in OLIVEIRA Ivone, SOARES Ana, Interfaces e Tendências da comunicação no contexto das organizações. São paulo: Difusão, 2008, p. 149177.

BALDISSERA Rudimar, Organizações como complexus de diálogos, subjetividades e significação. in Margarida Maria Krohling Kunsch. (Org.). A comunicação como fator de humanização das organizações. 1ed. São Caetano do Sul - SP: Difusão Editora, 2010, v. 3, p. 61-76.

BALDISSERA Rudimar, Comunicação organizacional, tecnologias e vigilância: entre a realização e o sofrimento. E-Compós (Brasília), v. 17, n. 2, p. 1-15, 2014.

BARROS Ellen, BRITO Joanicy, MACHADO Marlene, Interaçòes e Implicações paradigmáticas no contexto das organizações, in OLIVEIRA Ivone de L., PENNINI Anice, MOURÃO Isaura, Compreendendo um campo de conhecimento: reflexões epistemológicas sobre a comunicação Organizacional a partir de autores Brasileiros. Curitiba, PR:CRV, 2015

COLNAGO Camila Krohling, Os Novos Enfoques da Comunicação Organizacional no Ambiente Complexo. Texto apresentado no Congresso da Abrapcorp, 2007.

DEETZ Stanley, Comunicação Organizacional: fundamentos e desafios, in MARCHIORI M. (org.), Comunicação e Organização: reflexões, processos e práticas. São Caetano do Sul: Difusão, 2010, p. 83-101.

FREITAS Sidnéia Gomes, Comunicação interna e o diálogo nas organizações, in KUNSCH Margarida (org.), Gestão estratégica em Comunicação organizacional e relações públicas. São Paulo: Difusão, 2008, p. 139-153.

IASBECK Luis Carlos, Relações Públicas e Comunicação Organizacional; o lugar do texto e do contexto, Organicom, n. 10-11, 2009. 
KUNSCH Margarida M. K., Comunicação organizacional: conceitos e dimensões dos estudos e das práticas, in MARCHIORI M. (org.), Faces da cultura e da comunicação organizacional. São Paulo: Difusão, 2008, p. 169-192.

KUNSCH Margarida M. K., Percursos paradigmáticos e avanços epistemológicos nos estudos da comunicação organizacional, in KUNSCH M. (org.), Comunicação organizacional vol 1. São Paulo: Saraiva, 2009 vol. 1. p. 63 a 89.

KUNSCH Margarida M. K., Relações públicas e Comunicação organizacional: das práticas à institucionalização acadêmica, Organicom, n. 10-11, 2009.

LIMA Fábia, Possíveis contribuições do paradigma relacional para o estudo da comunicação no contexto organizacional, in OLIVEIRA Ivone Lourdes de; PAULA Carine F. Caetano de, Interfaces e tendências da comunicação no contexto das organizaçôes. São Paulo, Ed. Difusão, 2008.

LIMA Fábia, BASTOS Fernanda, Comunicação no contexto organizacional: afinal, o que é mesmo que estudamos? Trabalho apresentado ao GT Comunicación Organizacional e Relações Públicas do XXXI Congresso Brasileiro de Ciências da Comunicação/ Intercom - Natal, RN - 2 a 6 de setembro de 2008.

MARCHIORI Marlene, «A relação Comunicação-Organização: uma reflexão sobre seus processos e práticas». Texto apresentado no III Congresso da Abrapcorp, 2009. Disponível em: http://www.abrapcorp.org.br/anais2009/pdf/GT2_Marlene_Marchiori. pdf

MARQUES Ângela, Prefácio, in OLIVEIRA, Ivone de L; PENNINI Anice; MOURÃO Isaura, Compreendendo um campo de conhecimento: reflexões epistemológicas sobre a comunicação Organizacional a partir de autores Brasileiros. Curitiba, PR: CRV, 2015.

MAFRA Rennan, MARQUES Ângela, «Diálogo e organizações: cenas de dissenso e públicos como acontecimento», in MARQUES Ângela, OLIVEIRA Ivone de Lourdes. (orgs.), Comunicação Organizacional: dimensões epistemológicas e discursivas. Belo Horizonte: Fafich/ UFMG, 2015, p. 185-193.

MOL Vanessa, Contribuições da teoria dos sistemas sociais para a comunicação no contexto organizacional, in OLIVEIRA Ivone de L., PENNINI Anice, MOURÃO Isaura, Compreendendo um campo de conhecimento: reflexões epistemológicas sobre a comunicação Organizacional a partir de autores Brasileiros. Curitiba, PR:CRV, 2015

NASSAR Paulo, Conceitos e processos de comunicação organizacional, in KUNSCH Margarida (org.), Gestão estratégica em Comunicação organizacional e relações públicas. São Paulo: Difusão, 2008, p. 61-75.

OLIVEIRA Ivone de L., SOARES Ana Thereza N., Interfaces e tendências da comunicação no contexto das organizações. 2. ed. São Paulo, Ed. Difusão, 2011.

OLIVEIRA Ivone de Lourdes, Espaços dialógicos e relacionais nas organizações e sua natureza ético-política, in Margarida M. Krohling Kunsch. (Org.). Linguagem, gestão e perspectivas. São Paulo: Saraiva, 2009, v. 2, p. 321-332.

OLIVEIRA Ivone de Lourdes, Objetos de estudo da comunicação organizacional e das relações públicas: um quadro conceitual. Organicom, n. 10-11, 2009. 
PAULA Reynaldo Josué de, Inserção da racionalidade ético-comunicativa na práxis empresarial: possibilidades e limites. Revista Organizações \& Sociedade, v. 12, n. 35, 2005, p. 29-45.

PINTO Julio, Prefacio. Interfaces e tendências da comunicação no contexto das organizações. 2. ed. São Paulo, Ed. Difusão, 201. p. 9.

REIS Maria do Carmo, Comunicação Organizacional e Relações Públicas no Brasil são singulares? Organicom, n. 10-11, 2009.

RENAULT Emmanuel, L'expérience de l'injustice : reconnaissance et clinique de l'injustice. Paris, La Découverte, 2004.

ZANLUCHI Eliane, COPETTI Carmen, Comunicação Organizacional: desenvolvimento e consequências no contexto histórico das organizações. Texto apresentado no XXVII Congresso Brasileiro de Ciências da Comunicação, 2004.

WEBER Maria Helena, Comunicação Organizacional, a síntese. Relações Públicas, a gestão estratégica, Organicom, n. 10-11, 2009.

Résumé : L'objectif de cet article est de présenter la configuration des études en Communication Organisationnelle au Brésil. Seront ainsi évoquées les potentialités de ces recherches ainsi que les principaux problèmes qui se dressent devant les chercheurs qui œuvrent à la consolidation de ce champ. Une première difficulté est liée à la relation existant entre la Communication Organisationnelle et les Relations Publiques. Un deuxième problème concerne les tentatives de construction d'un corps conceptuel, cohérent et consistant, encore en émergence. Le troisième obstacle a trait à l'absence d'une problématisation scientifique intégrant la perspective critique, prenant en compte l'articulation des notions telles que politique, éthique, dialogue, conflits moraux, souffrance sociale, identité, discours, pouvoir et/ou pratiques de résistance. Malgré ces trois éléments, le texte met au jour les innombrables potentialités de croissance de ce champ, possibilités dont témoignent les recherches actuellement en cours dans nos Universités, laboratoires et groupes de recherche.

Mots clés : Communication Organisationnelle, Relations Publiques, Brésil, problèmes, potentialités, épistémologie.

Abstract: The aim of this article is to present the configuration of the Organizational Communication studies in Brazil. The potentialities of these researches will be evoked as well as the central problems that are faced by the researchers whose aim is to work on the consolidation of this field. A first difficulty is the complicated relation between organizational communication and public relations. A second problem is related to the attempts at construction of an emergent, coherent and consistent conceptual body. The third obstacle is the absence of a scientific problematization capable of integrating the critical perspective considering the articulation of concepts such as politics, ethics, dialogue, moral conflicts, social suffering, identity, discourses, power and/or practices of resistance. In spite of these three elements, the text highlights the various potentialities of expansion of this field largely testified by the researches currently developed in our Universities, laboratories and research groups.

Keywords: organizational communication, public relations, Brazil, problems, potentialities, epistemology. 